\title{
Stampede: A Cluster Programming Middleware for Interactive Stream-Oriented Applications
}

\author{
Umakishore Ramachandran, Rishiyur S. Nikhil, Member, IEEE, James M. Rehg, Yavor Angelov, \\ Arnab Paul, Sameer Adhikari, Kenneth M. Mackenzie, Nissim Harel, and Kathleen Knobe
}

\begin{abstract}
Emerging application domains such as interactive vision, animation, and multimedia collaboration display dynamic scalable parallelism and high-computational requirements, making them good candidates for executing on parallel architectures such as SMPs and clusters of SMPs. Stampede is a programming system that has many of the needed functionalities such as high-level data sharing, dynamic cluster-wide threads and their synchronization, support for task and data parallelism, handling of time-sequenced data items, and automatic buffer management. In this paper, we present an overview of Stampede, the primary data abstractions, the algorithmic basis of garbage collection, and the issues in implementing these abstractions on a cluster of SMPS. We also present a set of micromeasurements along with two multimedia applications implemented on top of Stampede, through which we demonstrate the low overhead of this runtime and that it is suitable for the streaming multimedia applications.
\end{abstract}

Index Terms-Middleware, cluster computing, streaming applications, garbage collection, virtual time.

\section{INTRODUCTION}

$\mathrm{E}$ MERGING application domains such as interactive vision, $\triangle$ animation, and multimedia collaboration display dynamic scalable parallelism and high-computational requirements, making them good candidates for executing on parallel architectures such as SMPs and clusters of SMPs. There are some aspects of these applications that set them apart from scientific applications that have been the main target of high performance parallel computing in recent years. First, time is an important attribute in such emerging applications due to their interactive nature. In particular, they require the efficient management of temporally evolving data. For example, a stereo module in an interactive vision application may require images with corresponding timestamps from multiple cameras to compute its output, or a gesture recognition module may need to analyze a sliding window over a video stream. Second, both the data structures as well as the producer-consumer relationships in such applications are dynamic and unpredictable at compile time. Existing programming systems for parallel computing do not provide the application programmer with adequate support for such temporal requirements.

To address these problems, we have developed an abstraction for parallel programming called Space-Time Memory (STM) - a dynamic concurrent distributed data structure for holding time-sequenced data. STM addresses the common parallel programming requirements found in most interactive applications, namely, intertask synchronization and meeting soft real-time constraints. These facilities are

- U. Ramachandran, J.M. Rehg, Y. Angelov, A. Paul, S. Adhikari, K.M Mackenzie, and N. Harel are with the College of Computing, Georgia Tech, 801 Atlantic Drive, Atlanta, GA 30332.

E-mail: $\{$ rama, rehg, yavor, arnab, sameera, kenmac, nissim\}@cc.gatech.edu.

- R.S. Nikhil is with Sandburst Corporation, 600 Federal Street, Andover 01810. E-mail: nikhil@sandburst.com.

- K. Knobe is with HP Labs-Cambridge Research Laboratory, One Cambridge Center, Cambridge, MA 02142. E-mail: kath.knobe@hp.com.

Manuscript received 8 Dec. 2002; revised 31 July 2003; accepted 3 Aug. 2003. For information on obtaining reprints of this article, please send e-mail to: tpds@computer.org, and reference IEEECS Log Number 118751. useful for this application class even on an SMP. However, in addition, our system provides the STM abstraction transparently across clusters. Reclamation of STM's time-sequenced data items is an unusual problem quite different from the usual memory address-based garbage collection. It is further complicated because the of the spread of the computation over a cluster. We present an algorithmic basis for automatic garbage collection across the cluster. We also discuss the issues in implementing these data abstractions on a cluster of SMPs.

STM was first implemented on a cluster of Alpha SMPs (running Digital Unix 4.0) interconnected by Memory Channel. Recently, we have ported Stampede to run on x86-Linux, StrongArm-Linux, and x86-NT platforms as well. We have used STM to implement the following applications so far:

1. a system for the analysis and synthesis of video textures which identifies transition points in a video sequence, and uses them to indefinitely extend the duration of a video clip,

2. a color-based vision tracking component for an interactive multimedia application called the Smart Kiosk, which was developed at the Compaq Cambridge Research Laboratory,

3. an image-based rendering application [8] at Compaq CRL, and

4. the distributed data management in an audio/video meeting application at Georgia Tech.

The key contributions of this paper are: ${ }^{1}$

- the presentation of the STM abstraction for parallel programming,

1. An overview of the Stampede programming system first appeared in a workshop [16]. The channel abstraction of Stampede and arguments of ease of use were presented in a conference [17]. Details of the garbage collection problem in Stampede were presented in another conference [15]. Discussion of the complete system details of Stampede and the performance study reported in this paper (Sections 6 and 7) have not appeared in any other forum. 


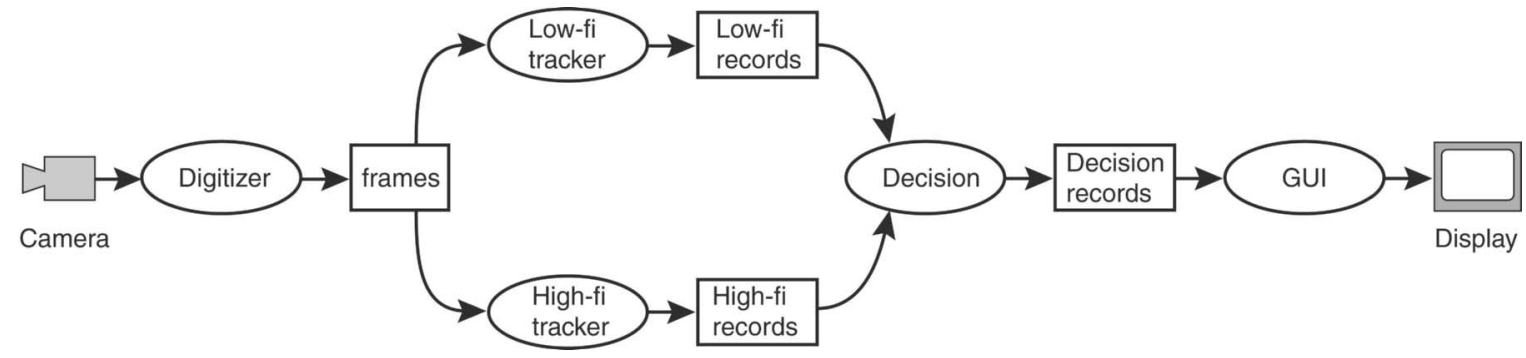

Fig. 1. A simple vision pipeline.

- a demonstration of ease of use in the context of programming interactive multimedia applications, and

- a performance study using this abstraction on a cluster of SMPs. In particular, we show that STM's significant programming advantage (over, say, direct message-passing) incurs only low performance overheads.

We begin by giving the application context in Section 2. In Section 3, we enumerate the parallel programming requirements engendered by interactive multimedia applications. The Space-Time Memory abstraction and the unusual garbage collection problem in this class of applications are discussed in Section 4. The ease of use of STM is demonstrated via programming examples in Section 4.7. We discuss design rationale in Section 4.8 and present related work in Section 4.9. A brief discussion of the implementation of Stampede is discussed in Section 5. Micromeasurements of the Stampede primitives, as well as application level studies using Stampede, are presented in Sections 6 and 7, and concluding remarks are given in Section 8.

\section{Application Context}

To set the context for the emerging application classes for which our cluster programming system is targeted, we briefly describe a new type of public computer device called the Smart Kiosk [25], [4], which has been developed at the Cambridge Research Laboratory of Compaq Computer Corporation. The Smart Kiosk could be located in public spaces such as a store, museum, or airport and is designed to interact with multiple people in a natural, intuitive fashion. For example, we envision Smart Kiosks that entertain passers-by while providing directions and information on local events. The kiosk may initiate contact with customers, greeting them when they approach and acknowledging their departure.

A Smart Kiosk may employ a variety of input and output devices for human-centered interaction: video cameras, microphones, infrared and ultrasonic sensors, loudspeakers, and touch screens. Computer vision techniques are used to track, identify, and recognize one or more customers in the scene [19]. A future kiosk will use microphone arrays to acquire speech input from customers and will recognize customer gestures. Synthetic emotive speaking faces [24] and sophisticated graphics, in addition to Web-based information displays, are currently used for the kiosk's responses.

We believe that the Smart Kiosk has features that are typical of many emerging scalable applications, including mobile robots, smart vehicles, intelligent rooms, and interactive animation. These applications all have advanced input/output modes (such as computer vision), very computationally demanding components with dynamic structure, and real-time constraints because they interact with the real world.

\section{Application Programming Requirements}

The parallel structure of the Smart Kiosk is highly dynamic. The environment in front of the kiosk (number of customers and their relative position) and the state of its conversation with the customers affect which threads are running, their relative computational demands, and their relative priorities (e.g., threads that are currently part of a conversation with a customer are more important than threads searching the background for more customers). There are a number of other applications (such as interactive animation and distributed audio/video meetings) that have similar characteristics to the Smart Kiosk.

A major problem in implementing these kinds of application is "buffer management." This is illustrated in the simple vision pipeline shown in Fig. 1. The digitizer produces digitized images every 30th of a second. The Low-fi tracker and the Hi-fi tracker analyze the frames produced by the digitizer for objects of interest and produce their respective tracking records. The decision module combines the analysis of such lower level processing to produce a decision output which drives the GUI that converses with the user. From this example, it should be evident that, even though the lowest levels of the analysis hierarchy produce regular streams of data items, four things contribute to complexity in buffer management as we move up to higher levels:

- Threads may not access their input data sets in a strict stream-like manner. In order to conduct a convincing real-time conversation with a human a thread (e.g., the Hi-fi tracker) may prefer to receive the "latest" input item available, skipping over earlier items. The conversation may even result in canceling activities initiated earlier so that they no longer need their input data items. Consequently, producer-consumer relationships are hints and not absolute, complicating efficient data sharing, especially in a cluster setting.

- Data sets from different sources need to be combined, correlating them temporally. For example, stereo vision combines data from two or more cameras, and stereo audio combines data from two or more microphones. Other analyzers may work 


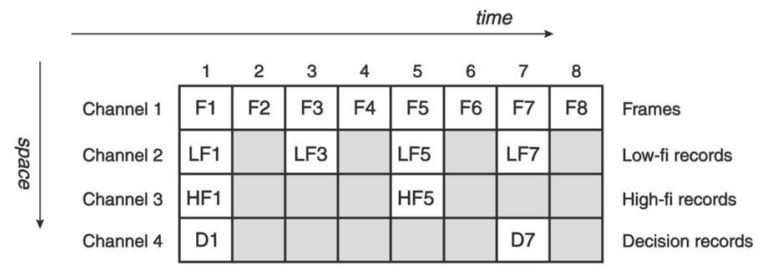

$=$ no record produced at this coordinate

Fig. 2. Mapping the vision pipeline to STM channels.

multimodally, e.g., by combining vision, audio, gestures, and touch-screen inputs.

- Newly created threads may have to reanalyze earlier data. For example, when a thread (e.g., a Low-fi tracker) hypothesizes human presence, this may create a new thread (e.g., a Hi-fi tracker) that runs a more sophisticated articulated-body or face-recognition algorithm on the region of interest, beginning again with the original camera images that led to this hypothesis. This dynamism complicates the recycling of data buffers.

- Since computations performed on the data increase in sophistication as we move through the pipeline, they also take more time to be performed. Consequently, not all the data that is produced at lower levels of the processing will necessarily be used at the higher levels. As a result, the data sets become temporally sparser and sparser at higher levels of processing because they correspond to higher and higher-level hypotheses of interesting events. For example, the lowest-level event may be: "a new camera frame has been captured," whereas a higherlevel event may be: "John has just pointed at the bottom-left of the screen." Nevertheless, we need to keep track of the "time of the hypothesis" because of the interactive nature of the application.

These algorithmic features bring up two requirements. First, data items must be meaningfully associated with time and, second, there must be a discipline of time that allows systematic reclamation of storage for data items (garbage collection).

In addition to the buffer management issue, specific tasks within these applications lend themselves very nicely to data parallelism. Consider, for example, the High-fi tracker in the vision pipeline shown in Fig. 1. The latency for processing a frame by this tracker could well exceed the rate at which the Digitizer (upstream) may produce frames for analysis. In such situations, an obvious approach would be to apply multiple processors to the tracking task operating in data parallel mode on distinct image frames or on parts of the same frame.

\section{Space-Time Memory}

The Stampede project addresses the parallel programming requirements posed by interactive multimedia applications such as those discussed in Section 2. Stampede allows the creation of multiple address spaces in the cluster and an unbounded number of dynamically created application threads within each address space. The threading model

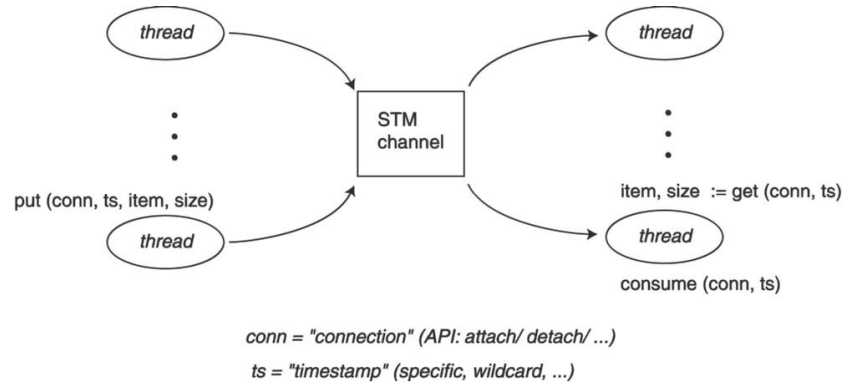

Fig. 3. Overview of Stampede channel usage (relationship of a channel to threads).

within an address space is basically standard OS threads such as pthreads (POSIX threads) on Tru64 Unix and Linux [6], and Win32 threads on Windows NT. Stampede provides high-level data sharing abstractions that allow threads to interact with one another without regard to their physical locations in the cluster or the specific address spaces in which they execute.

A novel component of Stampede is Space-Time Memory (STM), a distributed data structure that addresses the complex "buffer management" problem that arises in managing temporally indexed data items as in the Smart Kiosk application. Traditional data structures such as streams and lists are not sufficiently expressive to handle the requirements enumerated in the previous section.

STM channels provide random access to a collection of time-indexed data items, while STM queues give a FIFO access to a similar collection. We will first describe the channel and then remark on the similarities and differences between channels and queues. STM channels can be envisioned as a two-dimensional table. Each row, called a channel, has a system-wide unique id. A particular channel may be used as the storage area for an activity (e.g., a digitizer) to place the time-sequenced data records that it produces. Every column in the table represents the temporally correlated output records of activities that comprise the computation. For example, in the vision pipeline in Fig. 1, the digitizer produces a frame $F_{t}$ with a timestamp $t$. The Low-fi tracker produces a tracking record $L F_{t}$ analyzing this video frame. The decision module produces its output $D_{t}$ based on $L F_{t}$. These three items are on different channels and may be produced at different real times, but they are all temporally correlated and occupy the same column $t$ in the STM. Similarly, all the items in the next column of the STM channel table have the timestamp $t+1$. Fig. 2 shows an example of how the STM channels may be used to orchestrate the activities of the vision processing pipeline introduced in Fig. 1. The rectangular box at the output of each activity in Fig. 1 is an STM channel. The items with timestamp $1\left(F_{1}, L F_{1}, H F_{1}\right.$, and $D_{1}$ ) in each of the four boxes in Fig. 1 is a column in the STM.

\subsection{API to the STM Channel Abstraction}

The API has operations to create a channel dynamically, and for a thread to attach and detach a channel. Each attachment is known as a connection, and a thread may have multiple connections to the same channel. Fig. 3 shows an overview of how channels are used. A thread can put a data item into a channel via a given output connection using the call: 
spd_channel_put_item (o_connection, timestamp, buf_p, buf_size, ...).

The item is described by the pointer buf_p and its buf_size in bytes. A channel cannot have more than one item with the same timestamp, but there is no constraint that items be put into the channel in increasing or contiguous timestamp order. Indeed, to increase throughput, a module may contain replicated threads that pull items from a common input channel, process them, and put items into a common output channel. Depending on the relative speed of the threads and the particular events they recognize, it may happen that items are placed into the output channel out of order. Channels can be created to hold a bounded or unbounded number of items. The put call takes an additional flag that allows it to either block or return immediately with an error code if a bounded output channel is full.

A thread can get an item from a channel via a given connection using the call:

spd_channel_get_item (i_connection, timestamp,

\& buf_p, \& buf_size,

\& timestamp_range, ...).

The timestamp can specify a particular value, or it can be a wildcard requesting, for example, the newest/oldest value currently in the channel, or the newest value not previously gotten over any connection. As in the put call, a flag parameter specifies whether to block if a suitable item is currently unavailable, or to return immediately with an error code. The parameters buf_p and buf_size can be used to pass in a buffer to receive the item or, by passing NULL in buf_p, the application can ask Stampede to allocate a buffer. The timestamp_range parameter returns the timestamp of the item returned, if available; if unavailable, it returns the timestamps of the "neighboring" available items, if any.

The put and get operations are atomic. Even though a channel is a distributed data structure and multiple threads on multiple address spaces may simultaneously be performing operations on a given channel, these operations appear to all threads as if they occur in a particular serial order.

The semantics of put and get are copy-in and copy-out, respectively. Thus, after a put, a thread may immediately safely reuse its buffer. Similarly, after a successful get, a client can safely modify the copy of the object that it received without interfering with the channel or with other threads.

Puts and gets, with copying semantics are, of course, reminiscent of message-passing. However, unlike messagepassing, these are location-independent operations on a distributed data structure. These operations are one-sided: there is no "destination" thread/process in a put, nor any "source" thread/process in a get. The abstraction is one of putting items into and getting items from a temporally ordered collection, concurrently, not of communicating between processes.

\subsection{STM Queues}

The primary reason for providing the STM queue abstraction is to support data parallelism in a cluster. As we mentioned earlier, the targeted application classes provide plenty of opportunities for exploiting data parallelism. For example, in the vision pipeline (see Fig. 1), data parallel instances of the tracker could operate in parallel on distinct image frames or on parts of the same frame. STM queues are provided for this purpose. Similar to the channel, a queue has a system-wide unique id. The queue abstraction supports the same set of calls as a channel: get, put, and attach. The runtime system allows a "timestamp" attribute to be associated with an item in a queue just as in the case of a channel. Aside from the "timestamp" attribute associated with a queue item, the get/ put operations on a queue are semantically the same as the enqueue/dequeue operations on a traditional queue data structure. The nature of a traditional queue data structure coupled with the fact that the queue items have a timestamp attribute leads to the following differences between an STM queue and an STM channel:

- A get on a queue gives an item in strictly FIFO order (i.e., irrespective of the timestamp order of the queue items); the runtime provides the timestamp and ticket associated with this item to the getting thread.

- A queue item has to be gotten exactly once (otherwise, it will never go away as we will see in the next section) and cannot be gotten more than once; a channel item may be gotten zero times or as many times as the number of connections to that channel (modulo any reference count specification for that item, see Section 4.8).

- Multiple items with the same timestamp can be put into the queue; this may be necessary in a vision pipeline, for instance, if the strategy for data parallelism is to carve out a given image frame into smaller segments; the runtime system associates a tag (called a ticket) with each fragment that uniquely identifies a particular item in a queue.

\subsection{STM Registers}

In addition to channels and queues, Stampede also provides cluster-wide abstraction called registers. A thread can attach and detach to a register just like channels or queues. A register can be used like a cluster-wide shared variable. Writing to a register overwrites its previous contents. A register read operation returns successfully if a new value is written onto it. A thread can block on such a read until a new write happens. The full/empty synchronization semantics provides a mechanism to implement interthread signaling and event notification.

\subsection{Garbage Collection}

In dealing with timestamped data in this application domain, we encounter an unusual notion of garbage collection, where "reachability" concerns timestamps and not memory addresses. If physical memory were infinite, STM's put and get primitives would be adequate to orchestrate the production and access to time-sequenced data in any application. However, in practice, it is necessary to garbage collect data items that will no longer be accessed by any thread. When can we reclaim an item from a timestamp-indexed collection? The problem is analogous to the classical "space leak" situation where, whenever a table is reachable from the computation, no item in that table can be garbage collected on the basis of reachability alone, even if there are items that will never be accessed subsequently in the computation. A complication is the fact that 
application code can do arithmetic on timestamps. Timestamp-based GC is orthogonal to any classical addressbased GC of the STM's host language. This section discusses the guarantees provided by the STM for producing and accessing time-sequenced data, and the guarantees that the application must provide to enable garbage collection.

To enable garbage collection of an STM item, the API provides a consume operation by which the application declares to STM that a specific STM item ${ }^{2}$ is garbage from the perspective of a particular connection. A queue item has an implicit reference count of one. So, as soon as the thread that got that item calls consume on that item, STM can safely garbage collect it. Although get semantics is copyout as we shall see in Section 4.5, because of items that may involve embedded pointers, it is mandatory that consume be explicitly called. Garbage collection is a little more involved in the case of a channel. STM can safely garbage collect an item once it has determined that the item can no longer be accessed through any existing connection or any future connection to this channel. So, the discipline imposed by STM on the application programmer is to get an item from a channel, use it, and mark it as consumed. An object $X$ in a channel is in one of three states with respect to each input connection $i c$ attaching that channel to some thread. Initially, $X$ is "unseen." When a get operation is performed on $X$ over connection $i c$, then $X$ is in the "open" state with respect to $i c$. Finally, when a consume operation is performed on the object, it transitions to the "consumed" state. We also say that an item is "unconsumed" if it is unseen or open. The contract between the runtime system and the application is as follows: The runtime system guarantees that an item will not be garbage collected at least until it has been marked consumed on all the connections that have access to it. An application thread has to guarantee to mark each item on its input connections as consumed. The consume operation can specify a particular object (i.e., with a particular timestamp), or it can specify all objects up to and including a particular timestamp. In the latter case, some objects will move directly into the consumed state, even though the thread never performed a get operation on them.

Similarly, there are rules that govern the timestamp values that can be associated with items produced by a thread on a connection (be it a channel or a queue). A thread can associate a timestamp $t$ with an item it produces so long as this thread has an item $X$ with timestamp $t$ currently in the open state on one of its input connections. This addresses the common case (e.g., the Low-fi tracker thread in Fig. 1) where a thread gets an item from its input connection, processes it, produces a timestamped output (correlated to the timestamp of the item it is processing, possibly even the same timestamp) as a result of the processing, and marks the item consumed. We say that the output item inherits the timestamp of the input item.

However, there are situations where timestamped output may have to be generated without getting an item from the STM channel. This is, in general, true for application "source" threads that have no input connections (e.g., the digitizer thread in Fig. 1, with the corresponding code fragment shown in Fig. 6), or a root thread in a task

2. In the case of a channel, the (connection, timestamp) pair uniquely identifies a particular item. In the case of a queue, the (connection, ticket) pair uniquely identifies a particular item. connectivity graph that drives the whole computation. For this purpose, the STM maintains a state variable for each thread called virtual time. An application may choose any application-specific entity as the virtual time. For example, in the vision pipeline (Fig. 1), the frame number associated with each camera image may be chosen as the virtual time. The current visibility of a thread is the minimum of its virtual time and the timestamps of any items that it currently has open on any of its input connections. When a thread puts an item, it can give it any timestamp $\geq$ its current visibility. When a thread creates a new thread, it can initialize the child thread's initial virtual time to any value $\geq$ its own current visibility. When a thread creates a new input connection to a channel, it implicitly marks as consumed on that connection all items $<$ its current visibility. A thread can explicitly change its own virtual time to any value $\geq$ its current visibility. In most cases, a thread can set its own virtual time to the special value INFINITY because the timestamps of items it puts are simply inherited from those that it gets.

These rules enable the runtime system to transitively compute a global minimum $t s_{\text {min }}$, which is the minimum of:

- virtual times of all the threads,

- timestamps of all items on all queues, and

- timestamps of all unconsumed items on all input connections of all channels.

This is the smallest timestamp value that can possibly be associated with an item produced by any thread in the system. It is impossible for any current thread, or any subsequently created thread, ever to refer to an object with timestamp less than the global minimum. Thus, all objects in all channels with lower timestamps can safely be garbage collected. Stampede's runtime system has a distributed algorithm that periodically recomputes this value and garbage collects dead items. To ensure that this global minimum advances and, thus, garbage collection is not stymied, a thread must guarantee that it will advance its virtual time, for which STM provides an API call:

spd_set_virtual_time (new_virtual_time).

The consume call is reminiscent of reference counting. However, this gets complicated in the case of an STM channel because the number of consumers of an item is unknown-a thread may skip over items on its input connections and new connections can be created dynamically. These interesting and subtle issues, as well as our distributed, concurrent garbage collection algorithm are described in greater detail in a separate paper [15].

\subsection{Communicating Complex Data Structures through STM}

The put and get mechanisms described above are adequate for communicating contiguously allocated objects through channels and queues; but, what about linked data structures? In the Smart Kiosk vision code, for example, a "color model" data structure is actually a complex of four or more separately allocated components linked with $\mathrm{C}$ pointers. We wish to treat them as a single unit that can be communicated through STM. The $C$ pointers are, of course, meaningless in a different address space. 


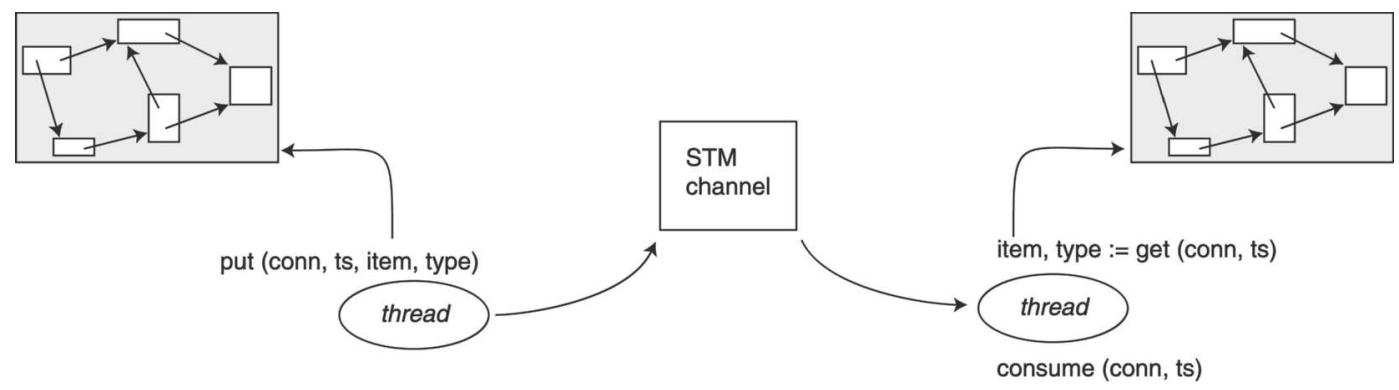

Fig. 4. Communicating complex objects through channels based on "types."

To solve this problem, Stampede extends the basic STM system with a notion of "object types." The following call:

spd_dcl_type (type, marshall_hook, unmarshall_hook,...),

declares a new object type (represented by an integer) with an associated set of methods or procedures. Two of these are hooks that assist in marshalling and unmarshalling objects for transmission between address spaces.

A variant of the channel/queue put procedure is based on types instead of object sizes. Its parameters include a pointer to the data structure and its type instead of its size (which is not particularly meaningful for a linked data structure). Similarly, a variant of the channel/queue get call returns a pointer to the linked data structure, and its type instead of size. Fig. 4 shows an overview of how these facilities are used. Stampede takes care of the marshalling, communication, and unmarshalling of the data structure, using the supplied hooks to decompose and reconstitute the "object." These actions are done lazily, i.e., only when a consumer actually attempts to get an item, and intermediate results are cached (at the producer and the consumers) to avoid repeating this work in the presence of multiple get's. The normal garbage collection process, described in the previous section, is extended to reclaim any such cached intermediate results.

If we implement Stampede in a language with a richer type system, the application programmer could perhaps be relieved of the burden of specifying these hooks (cf. "serializer" mechanisms in Java). However, even in this case, it would be useful to have the ability to override these default methods. For example, image data structures in the Smart Kiosk vision code include a linked list of attributes which can, in fact, be recomputed from the object during unmarshalling and, therefore, do not need to be transmitted at all. Further, the image data itself can be compressed during marshalling and decompressed during unmarshalling. Such application and type-specific generalizations of "marshalling" and "unmarshalling" cannot be provided automatically in the default methods.

In addition to serilization routines, the application can install specific garbage-handler routines to clean up such complex items. Although get has a copy-out semantics, it may copy out just a pointer to such an item. Therefore, typically, the runtime has no idea of when the item can be garbage collected. Only after an explicit consume call is made can the runtime run the installed routine to reclaim the memory.

\subsection{Synchronization with Real-Time}

The virtual time and timestamps described above with respect to STM are merely an indexing system for data items and do not in of themselves have any direct connection with real-time. For pacing a thread relative to real-time, Stampede provides an API for loose temporal synchrony that is borrowed from the Beehive system [22]. Essentially, a thread can declare real-time "ticks" at which it will resynchronize with real-time, along with a tolerance and an exception handler. As the thread executes, after each "tick," it performs a Stampede call attempting to synchronize with real-time. If it is early, the thread waits until that synchrony is achieved. It if is late by more than the specified tolerance, Stampede calls the thread's registered exception handler which can attempt to recover from this slippage. Using these mechanisms, for example, the digitizer in the vision pipeline can pace itself to grab images from a camera and put them into its output channel at 30 frames per second, using absolute frame numbers as timestamps.

\subsection{Programming Examples}

In this section, we show some STM programming examples. Fig. 5 shows the relationship of an application thread to the STM abstraction. The only interaction it has with the other threads in the application is via the Stampede data abstractions it is connected to on the input and output sides. Other than the specific calls to the STM to get, put, or consume an item, the thread executes its sequential algorithm.

For the vision pipeline in Fig. 1, we present code fragments for the digitizer thread and a tracker thread in Figs. 6 and 7, respectively.

It can be seen from the code fragments that the extent of application modification required to use the STM is small and localized to the specific regions where a thread would need to communicate with its peers under any parallel

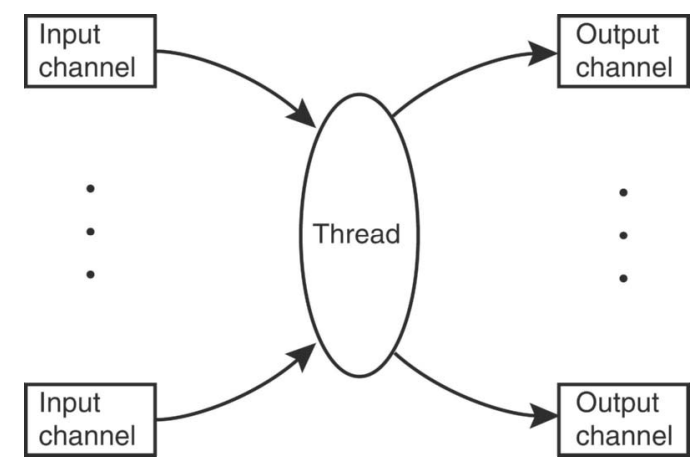

Fig. 5. Relationship of an application thread to STM. 


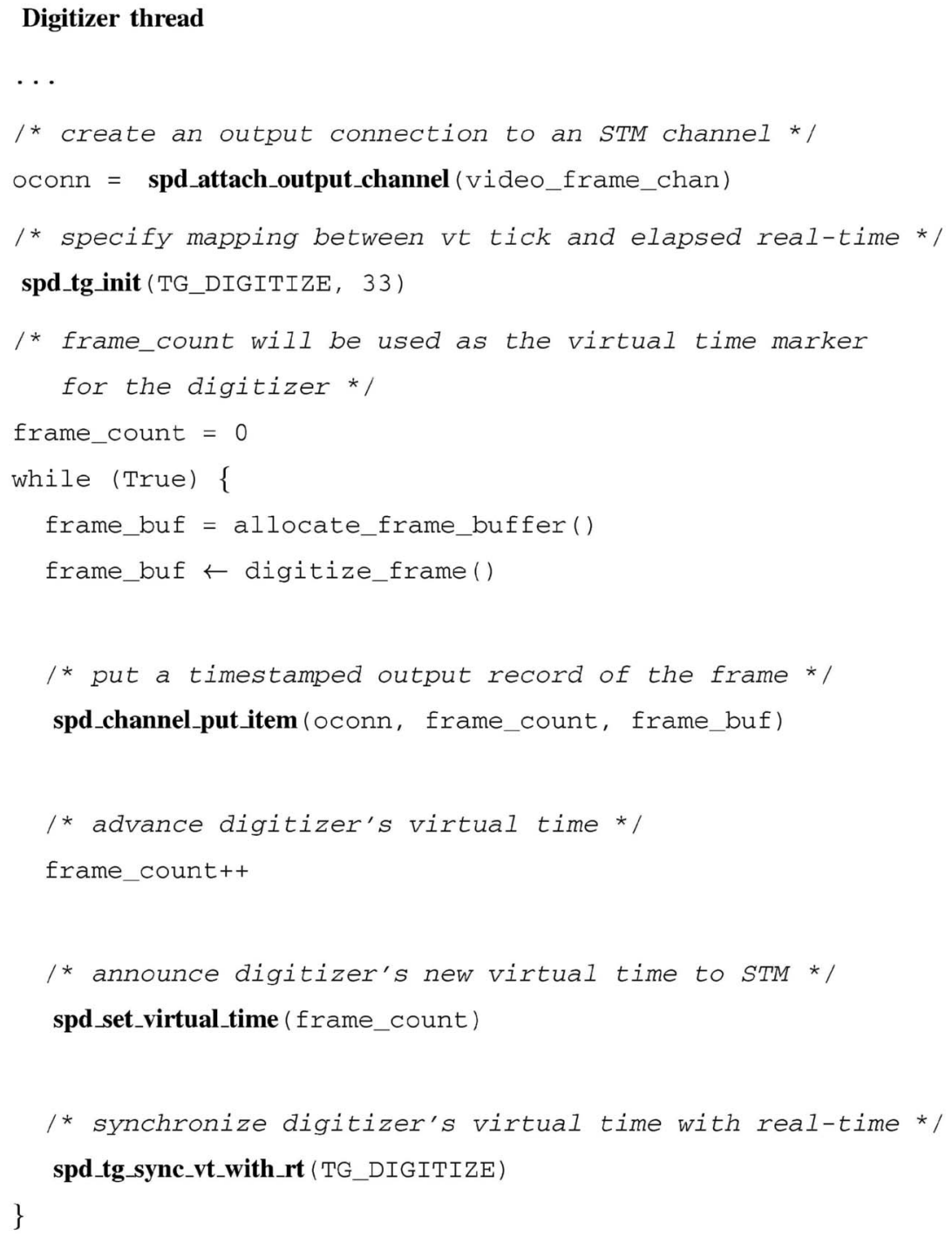

Fig. 6. Digitizer code using the STM calls.

programming regime. More importantly, all such communication and synchronization are encapsulated in the get, put, and consume calls. The threads never have to explicitly synchronize with other threads, nor do they have to know the existence of other threads in the applications. All that a particular thread needs to know is the names of the channels it should expect inputs from and the channels to which it should send its outputs (see Fig. 5). Thus, STM relieves the application programmer from low-level synchronization and buffer management. Moreover, the virtual time and timestamp mechanisms of the STM provide a powerful facility for the application programmer to temporally correlate disparate data items produced at different real-times by different threads in a complex application.

Space limitations prevent us from presenting more elaborate programming examples here. The program represented by the code fragments in Figs. 6 and 7 could perhaps have been written using straight message-passing, except that the STM code is still simpler because of its location-independence (producer and consumer need not be aware of each other), and because the consumer has the capability of transparently skipping inputs (using the STM_LATEST_UNSEEN flag in its get call). A more elaborate example would involve dynamic thread and channel creation, dynamic attachments to channels, multiple producers and consumers for a channel with complex production and consumption patterns, etc. These features, along with STM's automatic garbage collection, would be difficult to reproduce with message-passing code.

In addition to the Smart Kiosk system we have used throughout as a motivating example, Stampede is also being used in another application called image-based rendering [8], [20] at CRL. At Georgia Tech, it has been used to implement an audio/video meeting application, and a video texture generation application. A variant of the STM model has been investigated at Rice University for irregular scientific computations [3].

\subsection{Design Rationale}

In designing the STM abstraction, we have attempted to keep the interface simple and intuitive. We provide the reasoning behind some of the design choices we made along the way: 


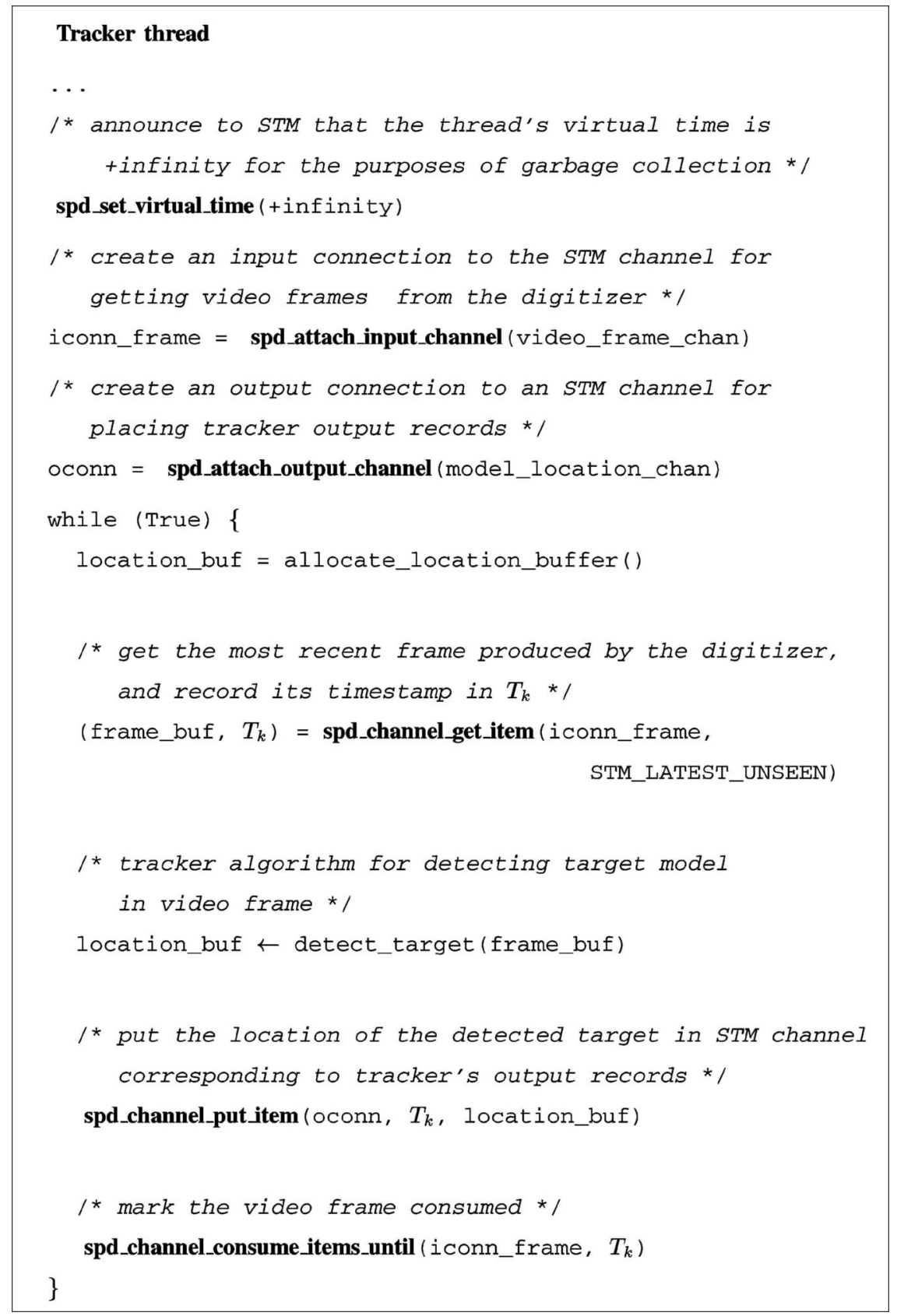

Fig. 7. Tracker code using the STM calls.

- Virtual versus Real Timestamps: Despite the fact that the primary intent of this abstraction is to support interactive applications, we chose an application-derived quantity to be used as timestamps. While some applications may benefit by using realtime for temporal correlation, it was not clear that, in general, the runtime could make correlations (using real-time) between independent streams that may use different sampling rates on input data (e.g., voice versus video). Further, data may be captured at some real-time but, processed at a much later realtime. By virtualizing time, the same timestamp index can be associated with both the raw and processed data, thus empowering applications to propagate temporal causality. We chose to allow the application to specify the mapping of the virtual time ticks to real-time and use that relationship purely for scheduling the threads (i.e., pacing an individual thread's activity) and not for temporal correlation.

There could be application scenarios in which there are streams (possibly with different time bases) that are semantically independent of one another from the application perspective. Clearly, the garbage collection of such streams should be independent of one another. However, garbage collection in Stampede relies on a single value, namely, the global virtual time. Thus, from the point of view of garbage collection, data items that are in such independent streams will be deemed by the runtime system as temporally related to one another if they happen to have the same timestamp. This is clearly a limitation of the current system. One possibility for circumventing this limitation is to define independent virtual time 
zones, and we will explore this possibility in future evolutions of the system.

- Virtual Time Management: As mentioned in Section 4.4, a "source" thread (with no input connections) must manage its virtual time explicitly, purely for the purpose of garbage collection, whereas most other threads implicitly inherit time based on what is available on their input connections. A more complex and contrived alternative would have been to let source threads make input connections to a "dummy" channel whose items can be regarded as "time ticks."

- Connections to Channels and Queues: A design choice is to allow operations directly on channels and queues instead of via explicit connections, thus simplifying the API. However, there are two reasons why we chose a connection-based access to channels and queues:

- $\quad$ The first reason has to do with flexibility. Our approach allows a thread to have multiple connections to the same channel. Such a flexibility would be valuable, for instance, if a thread wants to create a debugging or a monitoring connection to the same channel in addition to the one that it may need for data communication. While the same functionality could be achieved by creating a monitoring thread, we think that connections are a more intuitive and efficient way to achieve this functionality.

- The second reason has to do with performance. Connections can play a crucial role in optimizing communication especially in a cluster setting by providing a hint to the runtime system as to who may be potential consumers for a data item produced on a channel (so that data can be communicated early).

- Garbage Collection: STM provides transparent garbage collection by performing reachability analysis on timestamps. In a cluster, this could be quite expensive since the put and get operations on a channel or a queue are location transparent, and can be performed by threads anywhere in the cluster that have connections to that channel or queue. The alternative would have been to associate a reference count and garbage collect a channel item as soon as its reference count goes to zero. There are two issues with this alternative:

As we discussed earlier, not all produced items may necessarily be used in a dynamic application such as interactive vision. Thus, an item that was skipped over by all potential consumer threads will never be garbage collected since its reference count will never go to zero.

- Further, in many such dynamic applications, a producer may not know how many consumers there may be for an item it produces.

We do, however, allow a channel put operation to specify an optional reference count (a special value indicates that the consumer count is unknown to the producer). The runtime employs two different algorithms. The first algorithm uses reference counts. ${ }^{3}$ A second algorithm based on reachability

3. Recall that a queue item has an implicit reference count of one. analysis to garbage collect channel items with unknown reference counts is run less frequently.

\subsection{Related Work}

The STM abstraction may be viewed as a form of structured shared memory. In this sense, it is related to recent distributed shared memory systems (such as Cashmere [10], Shasta [21], and Treadmarks [9]). DSM systems typically offer the same API as any hardware SMP system and, therefore, are too low level to simplify programming of the complex synchronization and communication requirements found in interactive multimedia applications (as mentioned earlier, STM is useful, even on an SMP). Further, from a performance perspective, DSM systems are not particularly well-suited for supporting applications with highly dynamic sharing characteristics.

There have been several language designs for parallel computing such as Linda [1] (and its more recent derivatives such as JavaSpaces [12] and T-Spaces [26]), Orca [2], and Cid [13]. The data sharing abstractions in these languages are at a lower level than STM; of course, they could be used to implement STM.

Temporal correlation of independent data streams is a key distinguishing feature of our work from all prior work. The work most closely related to ours is the Beehive [22] software DSM system developed by one of the authors and his colleagues at the Georgia Institute of Technology. The delta consistency memory model of Beehive is well-suited for applications that have the ability to tolerate a certain amount of staleness in the global state information. Beehive has been used for real-time computation of computer graphical simulations of animated figures. STM is a higher level structured shared memory that can use the lower-level temporal synchronization and consistency guarantees of Beehive.

The idea of Space-Time memory has also been used in optimistic distributed discrete-event simulation [7], [5]. The purpose and, hence, the design of Space-Time memory in those systems is very different from ours. In those systems, Space-Time memory is used to allow a computation to rollback to an earlier state when events are received out of order. In this paper, we have proposed Space-Time Memory as the fundamental building block around which the entire application is constructed.

\section{IMPLEMENTATION}

Stampede was originally implemented (at Compaq CRL) on a cluster of 4-way Alpha SMPs interconnected by Memory Channel and running Tru64 Unix. Since then, it has been ported to clusters of x86-Linux, x86-Solaris, StrongArmLinux, and Windows nodes. The Stampede runtime systems assumes a reliable messaging layer underneath. We have built Stampede on top of two such messaging layers: MPI [11] and CLF [14]. Both MPI and CLF provide basic message transport. While MPI uses TCP/IP for reliability, CLF implements its own packet-based reliability layer on top of UDP. Stampede facilitates the creation of any number of address spaces in each node of the cluster, and threads within each address space. The channels/queues can be created in any of the address spaces and have system-wide unique ids allowing transparent access to them by a thread running anywhere in the cluster. The runtime implements caching of items fetched from remote channels and queues for transparent sharing by Stampede threads collocated on the same node of the cluster. Detailed 
discussion of the implementation details of the Stampede runtime library is beyond the scope of this paper.

\section{Baseline Performance of Stampede}

In addition to simplifying programming, STM has the potential to provide good performance on clusters for several reasons. First, synchronization and data transfer are combined in STM, permitting fewer communications. Second, connections provide useful hints for optimizing data communication across clusters. Third, sharing across address spaces is orchestrated via the STM abstraction, which can therefore optimize it in a more targeted manner than the indiscriminate sharing that can occur in a DSM system for dynamic applications.

We have conducted two sets of experiments to evaluate the performance of Stampede: first, a set of microbenchmarks and, next, a set of applications. In the microbenchmarking experiments, we measured the latency and bandwidth of put/get calls in Stampede. Comparison of the latencies taken by the Stampede calls against the latencies taken by the messaging layer underneath reveals that STM incurs only nominal marginal processing cost. Similarly, recording the sustained bandwidth at the STM runtime level reveals that STM offers bandwidth comfortably above what is required for continuous display of moderate resolution camera images at 30 frames/second. Due to space restrictions, we provide detailed description of the experiments and the results in Appendix A. "Appendix A can be found on the Computer Society Digital Library at http:/ / computer.org/ tpds/archives.htm."

In Section 7, we present performance studies for representative multimedia applications implemented using Stampede.

The computing platform for all the experiments is a cluster of SMP nodes running Linux. The hardware consists of 17 Dell 8450 servers, each with eight $550 \mathrm{MHz}$ Pentium III Xeon CPUs, $2 \mathrm{MB}$ of L2 cache per CPU, and 4GB of memory per node. The 8450 uses the Intel ProFusion chipset which provides two 64-bit/100MHz system (front-side) busses, one for each bank of four CPUs. The nodes are interconnected with doubled Gigabit Ethernet through a dedicated switch.

\section{Application-Level Performance}

In this section, we describe a set of experiments for evaluating the performance of two interactive multimedia applications on the Stampede cluster. The first is a video textures application. This application takes an input video sequence and calculates a set of cut points which can be used to "loop" the video sequence indefinitely, by randomly transitioning between the computed cuts. This requires calculating the best matches across all pairs of frames in order to identify the best places to cut. This is a computationally-demanding batch video processing task.

The second application is a color tracker that operates on a live video stream and tracks the locations of multiple moving targets on the basis of their color signatures. This application requires real-time color analysis of the video frames and comparison against multiple color models. These two applications are typical of the kinds of computations that new multimedia systems will require. They exhibit an interesting range of computational properties, which are illustrated in the experiments that

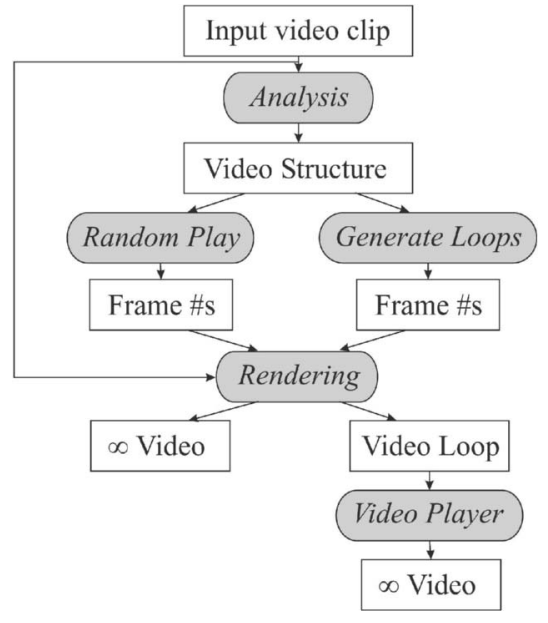

Fig. 8. Video Texutre System Overview. An input video clip is fed into the Analysis component, which finds good transition points where the video can be looped back on itself. These transitions (the Video Structure) are fed to one of two Synthesis components: either Random Play, which sequences the transitions stochastically, or Generate Loops, which finds a set of transitions that together create a single overall video loop of a given length. The Rendering component takes the generated sequence of frames, together with the original video clip, and produces either an infinite video texture sequence, or a video loop that can be played indefinitely by a standard Video Player in "loop" mode.

follow. Due to space restriction, we present the second application and its performance in Appendix B. "Appendix B can be found on the Computer Society Digital Library at http://computer.org/tpds/archives.htm."

The software is Linux-based with Intel-provided libraries. The operating system is Linux with the 2.4.9 kernel. The system scheduler in this kernel is oblivious to the 8450 's split system bus. The compiler is GCC version 2.96 with optimization set to -O2. The video textures application uses the ippiNormDiff_L2_8u_C1R() procedure in Intel's Integrated Performance Primitives (IPP) library, version 1.1 at the core of its processing. For these application level studies, we use Stampede on top CLF.

\subsection{Experiments with Video Textures}

The overall application pipeline is shown in Fig. 8. The computationally intensive part of the application is the box labeled Analysis. This represents the kernel of the application that we parallelize on the cluster. The distinguishing characteristic of this application kernel is that the input data set of $N$ images are all produced by a digitizer thread that is on one node of the cluster (the images come from one camera source which can be thought of as attached to that cluster node). The core computation in the kernel is a comparison of every image with every other image in the sequence. Thus, the total work to be done in the kernel is the total number of image comparisons: $W=N(N-1) / 2$. The actual computation is an L2 differencing ${ }^{4}$ between every two images. Note that every pair of image comparison is independent of other comparisons. In this sense, the kernel is embarrassingly parallel. The tricky part of parallelizing this kernel is determining an efficient partitioning scheme to minimize the data distribution costs,

\footnotetext{
4. The L2 norm of the difference of two vectors $u$ and $v$ is: $\sqrt{\operatorname{sum}\left((u-v)^{2}\right)}$. Each image is "unwrapped" in raster scan order to form a vector.
} 


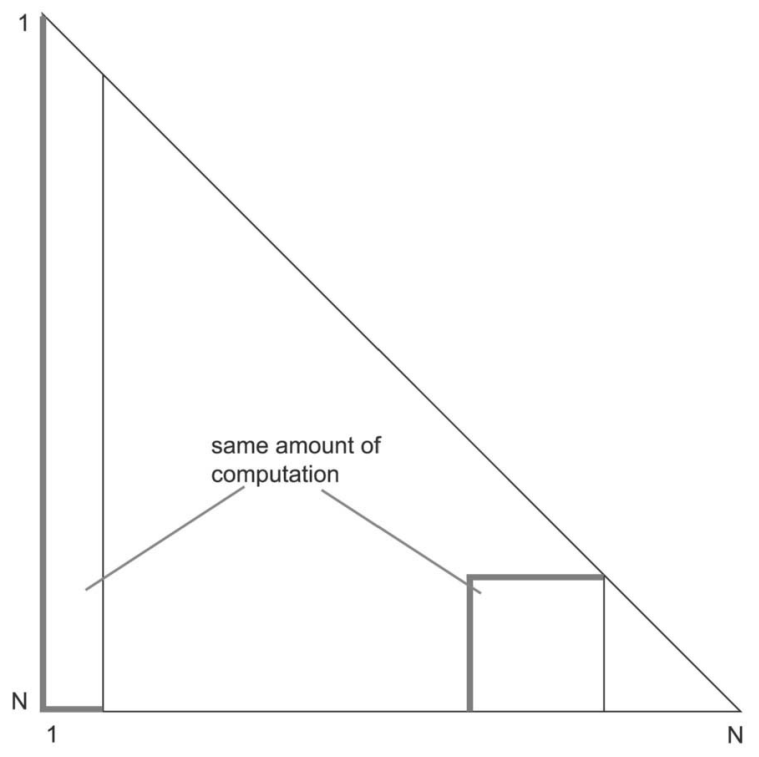

Fig. 9. Video Textures Computation Space: The lower triangular nature of the computation space has a direct impact on choice of work distribution. The column on the left and the square tile on the right perform an equal number of image comparisons. However, the column would require fetching all $N$ images from the digitizer for comparison against one image, while the square tile requires only a subset of images and reuses them.

improve cache performance and, thus, optimize the overall execution time on the cluster.

Fig. 9 shows the input data set and the computation space of the kernel. In the experimental study, we use a total of 316 images amounting to a total of 49,770 image comparisons. Each image is of size $640 \times 480$ color pixels, approximately $900 \mathrm{~KB}$.

\subsubsection{Mapping the Video Texture Analysis onto Stampede}

We use the Stampede programming library to implement the video texture analysis kernel. Fig. 10 shows the implementation using Stampede threads, channels, and queues. AS0, AS1, ..., ASN denote the cluster nodes. Stampede threads within the same node share memory. The digitizer thread, a channel for image distribution, a queue for collecting the correlation results, and a completion recognizer thread that is notified when the analysis is complete are all located on AS0. Each of the other cluster nodes participating in the computation has one data mover thread and some number of worker threads. The data mover thread prefetches images from the image distribution channel and, depending on the data distribution scheme (to be discussed shortly), may also pass them around to other nodes of the cluster via Stampede channels. The worker threads carry out the image comparisons. The digitizer gives sequential numbers as the "timestamp" for an image that is put on a channel, and the data mover and worker threads use this timestamp to get a particular image from the channel. The timestamp essentially serves as an index into the image array that is contained in the image distribution channel. This is an interesting and unintended use of the Stampede timestamp mechanism and has two consequences. The first is a programming convenience in that the channel appears logically like shared memory across the cluster nodes. The second is a performance consequence in that an image that is prefetched into a cluster node by the data mover thread is shared by all the worker threads that are on this node via the Stampede get operation, since Stampede runtime caches items that are fetched from remote nodes. Stampede "registers" are used for event notification purposes (not shown in the figure) and to exchange global information.

\subsubsection{Performance Concerns}

While it is straightforward to implement the video texture kernel using Stampede, it is nontrivial to optimize the kernel for performance. To address the performance concerns related to internode distribution of data, we introduce a work distribution strategy similar to multicast on top of unicast (Fig. 11). To address memory hierarchy performance concerns (particularly cache hit ratio), we compare images in stripes of pixels, rather than as a whole, and apply heuristics to order the memory requests.

\subsubsection{Experimental Setup}

The input data set is 316 images of $900 \mathrm{~KB}$ each. We conducted the following experiments:

- $\quad$ Stripe size. The intent is to determine the speedup on a single node with varying stripe size and number of worker threads.

Even with a single worker thread, we would expect performance improvement as the stripes become smaller since the memory bus may not be able to serve even a single CPU doing full-size image comparison. The experiments included varying the number of worker threads from one to eight, and the size of stripes from two to 480 lines of pixels, where each line is 1,920 bytes. The optimum stripe size is nonobvious since the intermediate results have to be cached and later combined.

- Efficiency of internode distribution strategy. This experiment is intended to test the scalability of the

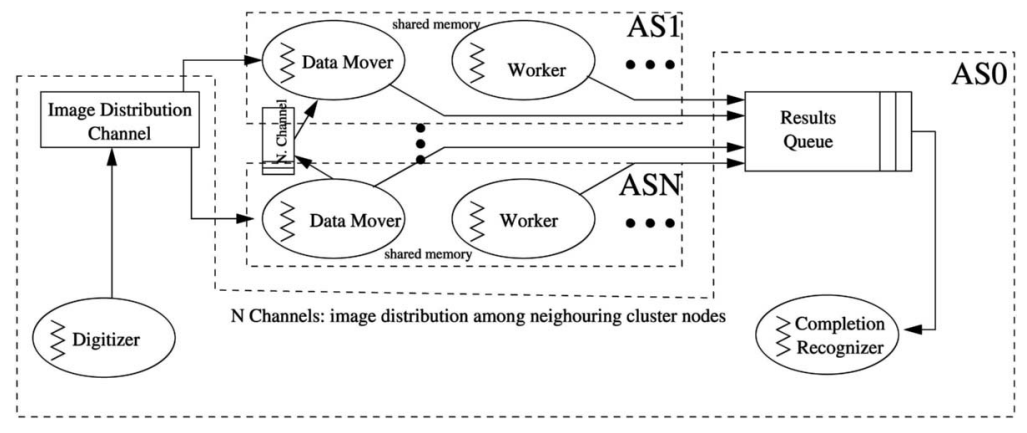

Fig. 10. Video texture implementation. 


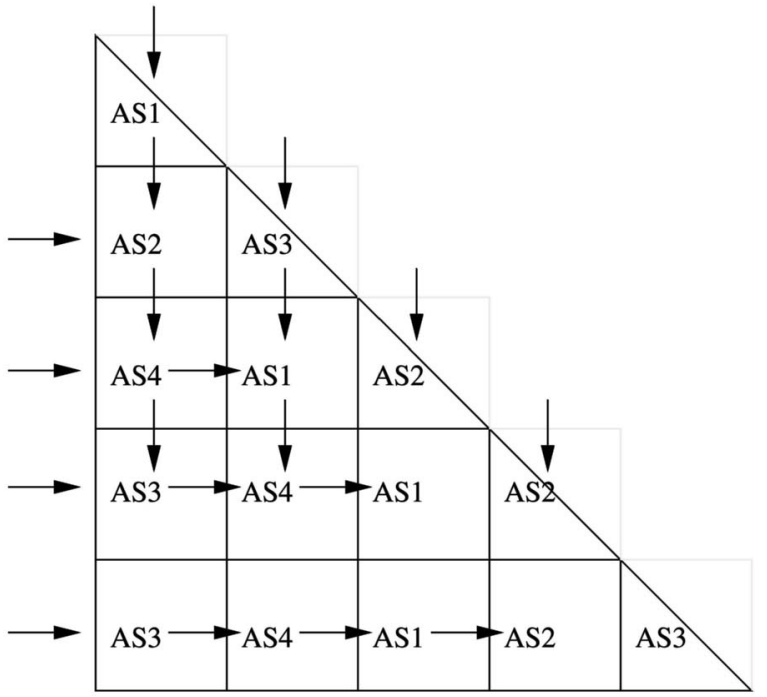

Fig. 11. Conceptual diagram of the tiling-with-chaining work distribution strategy.

internode distribution strategy. Each node employs a single worker thread. The number of nodes is increased from two to 14, with node AS0 serving as the source node for the images.

- Overall speedup. This experiment is intended to assess the overall speedup as the number of nodes is increased from two to 14, and the number of worker threads is increased from one to eight.

\subsubsection{Results and Discussion}

Fig. 12 shows the results of the stripe-size experiment. The curve for a stripe size of 480 lines represents fetching entire images; the execution time flattens out at about 27 seconds (a speedup of about 3.5) corresponding closely to the memory bandwidth limit of the 8450 system.

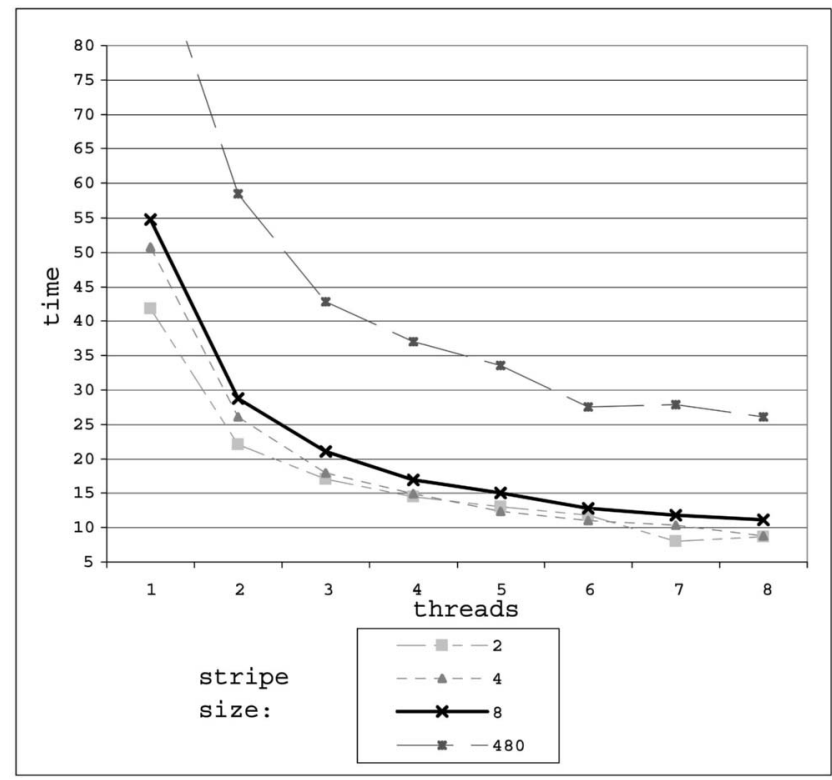

Fig. 12. Running times (seconds) for different configurations of stripe sizes and number of threads. A stripe size of 2 corresponds to performing comparisons one line of pixels ( 3,840 bytes) at a time.

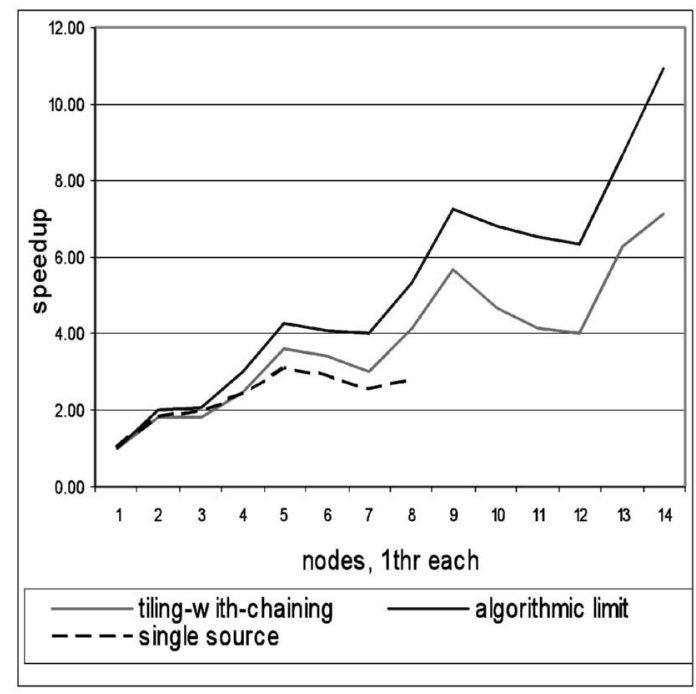

Fig. 13. Speedup across nodes for one thread per node.

The other three curves represent different choices in stripe sizes intended to fit in the cache and the results reveal an optimum stripe size of two. The results for stripe size of four comes close which, at first glance, is surprising since the worst-case cache capacity needed for this stripe size is $2,370 \mathrm{~KB}(4 \times 1,920 \times 316)$, which is more than the available cache size of $2 \mathrm{MB}$. However, due to the incomplete tiles along the edges of the triangle the average cache size needed is much less than the worst case leading to this result. The best speedup achieved is 5.75 for eight workers, which is pretty good considering that there is a data mover thread in addition to the workers taking up some cycles on the node.

Clearly, the optimum stripe size is a function of the problem size (i.e., the number of images being compared) and the available cache on the processor. With smaller tile sizes and appropriately chosen stripe sizes, machines with smaller cache sizes will be able to minimize the memory bottleneck.

In Figs. 13 and 14, we show speedup results for the networked distribution and for the single-source distribu-

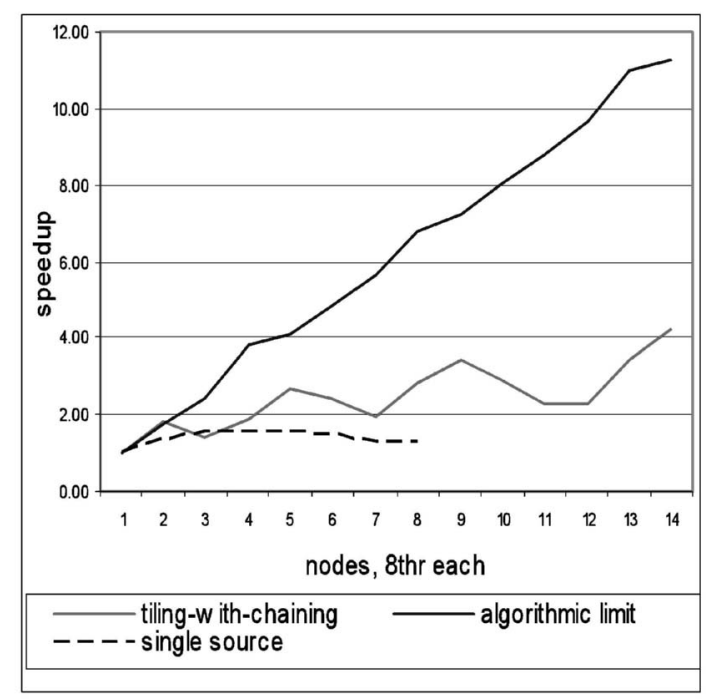

Fig. 14. Speedup across nodes for eight threads per node. 


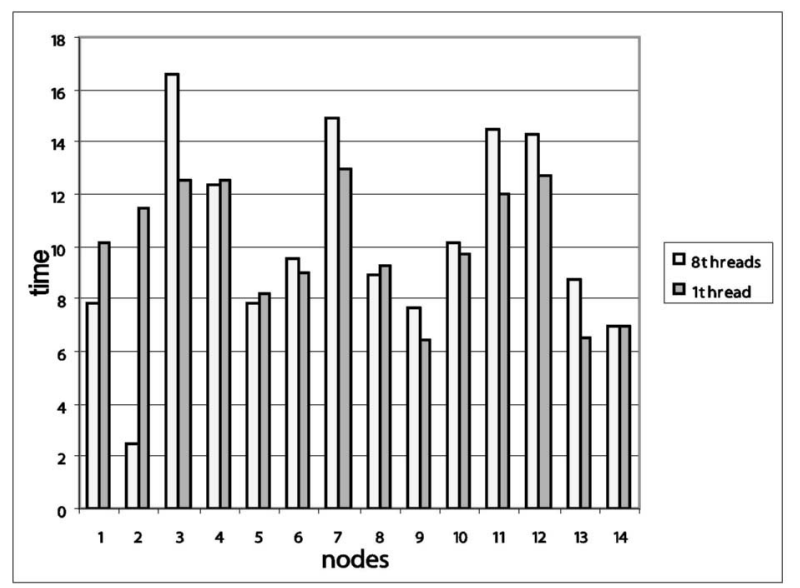

Fig. 15. Internode communication (in seconds). Time spent by each thread in network $\mathrm{I} / \mathrm{O}$ for 1 -thread and 8-thread per node configurations as we vary the number of nodes from one to 14 .

tion. For these results, we have not used cache stripe optimization, but compare full images. As the number of nodes are increased, the single source becomes a bottleneck limiting scalability. This is understandable since with a problem size of 316 images, the single source has to pump in the worst case $278 \mathrm{MB}$ to each node of the cluster. Of course, with tiling, the actual number of images needed by each node is smaller than this worst case. The maximum delivered bandwidth we have observed using Stampede is $45 \mathrm{MB} / \mathrm{s}$ on Gigabit Ethernet. Thus, with 14 nodes, it would take $\approx 100 \mathrm{sec}$ just for data distribution in the worst case.

As a point of comparison, the entire computation (without the cache stripe optimization) takes about $90 \mathrm{~s}$ using a single CPU on a single node.

Figs. 13 and 14 also show the ideal algorithm-limited speedup [23] due to the load imbalance inherent in our choice of tiles. Recall that the tile size decreases with the number of nodes. As can be seen, the bigger the tiles, the better the compute : communication ratio, yet the more jagged but steep is the algorithmic speedup curve. Because the algorithmic speedup accounts only for load balancing but not communication costs, if the tile size is 1, the algorithmic speedup curve will be a 45 -degree line, since work can be distributed perfectly evenly. Needless to say, this tile size is also the worst possible in terms of communication since all the nodes will need all the images.

The line labeled tiling-with-chaining shows the overall performance in the presence of networked distribution. With one worker per node (Fig. 13), we observe speedup of about 7.1 for 14 nodes. As can be seen, the corresponding algorithmic limit is 10.9. For eight workers per node, we observe speedup of about 4.22 (Fig. 14), while the algorithmic limit is 11.29.

Since the base with eight threads had a speedup of 5.75 , that translates to an overall speedup of 24.26 on 112 processors.

We attribute the difference between 1-thread and 8-thread performance ( 7.1 versus 4.22 ) to communication costs. Fig. 15 compares the communication costs for one and eight threads as we vary the number of nodes in the application. As can be seen, each thread in the 8-thread configuration spends roughly the same amount of time as the singleton thread in the 1-thread configuration on network $\mathrm{I} / \mathrm{O}$, leading to a reduction in overall speedup. The communication time is relatively stable (due to the application-level multicast distribution tree) instead of increasing, as is the case with a single source.

With some detailed instrumentation of the Stampede system, we have been able to ascertain that most of the communication inefficiency is limited to prefetching the initial tile. During this phase, there can obviously be no overlap of computation with communication. This effect is exacerbated with eight workers per node over the effect with one worker per node and, hence, the corresponding disparity in the speedup curves. However, once the initial tile has been brought in, there is good overlap of computation and communication due to the data mover thread at each node.

\section{Concluding Remarks}

Stampede is a cluster parallel programming system with novel data abstractions designed to support emerging classes of complex interactive stream-oriented multimedia applications. Space-time memory (with its two variants channel and queues) provides a rich high level programming support to alleviate the programmer from low level details in developing such applications on a cluster computing platform. There are nontrivial systems issues in implementing this abstraction (most notably the garbage collection problem) efficiently in a cluster. We presented the details of the STM abstraction, programming examples to demonstrate its ease of use, and performance studies on a cluster of SMPs to show the implementation efficiency.

Directions for future research include asynchronous notification of item arrival on channels and queues, and multicasting support at the level of the abstractions.

\section{ACKNOWLEDGMENTS}

A number of people have contributed to the Stampede project. Bert Halstead, Chris Jeorg, Leonidas Kontothanassis, and Jamey Hicks contributed during the early stages of the project. Dave Panariti developed a version of CLF for Alpha Tru64 and Windows. Members of the "ubiquitous presence" group at Georgia Tech continue to contribute to the project: Bikash Agarwalla, Matt Wolenetz, Hasnain Mandviwala, Phil Hutto, Durga Devi Mannaru, Namgeun Jeong, and Ansley Post deserve special mention. Russ Keldorph and Anand Lakshminarayanan developed an audio and video meeting application. Irfan Essa and Arno Schoedl provided the background necessary for understanding the video texture application. This work was done while R.S. Nikhil was at Compaq CRL. This work has been funded in part by a US National Science Foundation ITR grant CCR-01-21638, US National Science Foundation grant CCR-99-72216, HP/Compaq Cambridge Research Lab, the Yamacraw project of the State of Georgia, and the Georgia Tech Broadband Institute. The equipment used in the experimental studies is funded in part by a US National Science Foundation Research Infrastructure award EIA-9972872, and Intel Corp.

\section{References}

[1] S. Ahuja, N. Carriero, and G. David, "Linda and Friends," Computer, vol. 19, no. 8, pp. 26-34, Aug. 1986.

[2] H.E. Bal, A.E. Tanenbaum, and M.F. Kaashoek, "Orca: A Language for Distributed Programming," ACM SIGPLAN Notices, vol. 25, no. 5, pp. 17-24, May 1990. 
[3] A. Chauhan and K. Knobe, "A Compiler Driven Execution Model for Irregular Applications," Proc. Workshop Compilers for Parallel Computers, Jan. 2000.

[4] A.D. Christian and B.L. Avery, "Digital Smart Kiosk Project," Proc. ACM SIGCHI, pp. 155-162, Apr. 1998.

[5] K. Ghosh and R.M. Fujimoto, "Parallel Discrete Event Simulation Using Space-Time Memory," Proc. 20th Int'l Conf. Parallel Processing, Aug. 1991.

[6] IEEE, Threads Standard POSIX 1003. 1c-1995 (also ISO/IEC 99451:1996), 1996.

[7] D.R. Jefferson, "Virtual Time," ACM Trans. Programming Languages and Systems, vol. 7, no. 3, pp. 404-425, July 1985.

[8] S.B. Kang, "A Survey of Image-Based Rendering Techniques," Technical Report CRL 97/4, Cambridge Research Lab., Digital Equipment Corp., Aug. 1997.

[9] P. Keleher, A. Cox, S. Dwarkadas, and W. Zwaenepoel, "TreadMarks: Distributed Shared Memory on Standard Workstations and Operating Systems," Proc. Winter Usenix, 1994.

[10] L. Kontothanassis, G. Hunt, R. Stets, N. Hardavellas, M. Cierniak, S. Parthasarathy, W. Meira, S. Dwarkadas, and M. Scott, "VMBased Shared Memory on Low-Latency Remote-Memory-Access Networks," Proc. Int'l Symp. Computer Architecture, June 1997.

[11] Message Passing Interface Forum, MPI: A Message-Passing Interface Standard, www.mpi-forum.org, May 1994.

[12] S. Microsystems Javaspaces Specification, http://java.sun.com/ products/javaspaces, 1998.

[13] R. S. Nikhil, "Cid: A Parallel Shared-Memory C for Distributed Memory Machines," Proc. Seventh Ann. Workshop Languages and Compilers for Parallel Computing, pp. 376-390, Aug. 1994.

[14] R.S. Nikhil and D. Panariti, "CLF: A Common Cluster Language Framework for Parallel Cluster-Based Programming Languages," technical report (forthcoming), Digital Equipment Corp., Cambridge Research Laboratory, 1998.

[15] R.S. Nikhil and U. Ramachandran, "Garbage Collection of Timestamped Data in Stampede," Proc. 19th Ann. Symp. Principles of Distributed Computing, July 2000.

[16] R.S. Nikhil, U. Ramachandran, J.M. Rehg, R.H. Halstead, Jr., C.F. Joerg, and L. Kontothanassis, "Stampede: A Programming System for Emerging Scalable Interactive Multimedia Applications," Proc. 11th Int'l Workshop Languages and Compilers for Parallel Computing, Aug. 1998.

[17] U. Ramachandran, R.S. Nikhil, N. Harel, J.M. Rehg, and K. Knobe, "Space-Time Memory: A Parallel Programming Abstraction for Interactive Multimedia Applications," Proc. ACM Principles and Practices of Parallel Programming, May 1999.

[18] J.M. Rehg, K. Knobe, U. Ramachandran, R.S. Nikhil, and A. Chauhan, "Integrated Task and Data Parallel Support for Dynamic Applications," Scientific Programming, vol. 7, nos. 3-4, pp. 289-302, 1999.

[19] J.M. Rehg, M. Loughlin, and K. Waters, "Vision for a Smart Kiosk," Computer Vision and Pattern Recognition, pp. 690-696, June 1997.

[20] J.M. Rehg, U. Ramachandran, R.H. Halstead, Jr., C. Joerg, L. Kontothanassis, and R.S. Nikhil, "Space-Time Memory: A Parallel Programming Abstraction for Dynamic Vision Applications," Technical Report CRL 97/2, Digital Equipment Corp., Cambridge Research Lab, Apr. 1997.

[21] D.J. Scales, K. Gharachorloo, and C.A. Thekkath, "Shasta: A Low Overhead, Software-Only Approach for Supporting Fine-Grain Shared Memory," Proc. Seventh ASPLOS,, Oct. 1996.

[22] A. Singla, U. Ramachandran, and J. Hodgins, "Temporal Notions of Synchronization and Consistency in Beehive," Proc. Ninth Ann. ACM Symp. Parallel Algorithms and Architectures, June 1997.

[23] A. Sivasubramaniam, A. Singla, U. Ramachandran, and H. Venkateswaran, "An Application-Driven Study of Parallel Systems Overheads and Network Bandwidth Requirements," IEEE Trans. Parallel and Distributed Systems, Mar. 1999.

[24] K. Waters and T. Levergood, "An Automatic Lip-Synchronization Algorithm for Synthetic Faces," Multimedia Tools and Applications, vol. 1, no. 4, pp. 349-366, Nov. 1995.

[25] K. Waters, J.M. Rehg, M. Loughlin, S.B. Kang, and D. Terzopoulos, "Visual Sensing of Humans for Active Public Interfaces," Computer Vision for Human-Machine Interaction, R. Cipolla and A. Pentland, eds., pp. 83-96, Cambridge Univ. Press, 1998.

[26] P. Wyckoff, S. McLaughty, T. Lehman, and D. Ford, "T Spaces," IBM Systems J., vol. 37, no. 3, pp. 453-474, Aug. 1998.

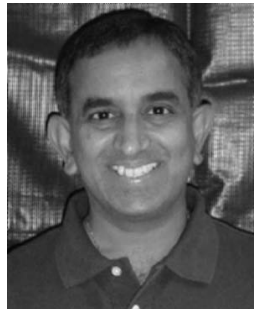

Umakishore Ramachandran received the $\mathrm{PhD}$ degree in computer science from the University of Wisconsin, Madison, in 1986, and is currently a professor in the College of Computing at the Georgia Institute of Technology. His fields of interest include parallel and distributed systems, computer architecture, and operating systems. Currently, he is leading a US National Science Foundation-ITR funded project that investigates the programming idioms and runtime systems for a distributed sensing infrastructure. He is the recipient of US National Science Foundation PYI Award in 1990, the Georgia Tech doctoral thesis advisor award in 1993, and the College of Computing Outstanding Senior Research Faculty award in 1996.

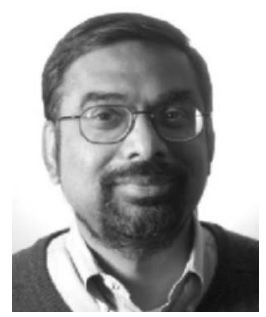

Rishiyur S. Nikhil received the PhD degree from the University of Pennsylvania. He has been at Sandburst Corporation since September 2000, directing the development of Bluespec (TM), a new language for ASIC design and verification. Previously, he was at the Cambridge Research Laboratory (DEC/Compaq), including a brief stint as acting director. Earlier, he was an associate professor of computer science and engineering at Massachusetts Institute of Technology. He has published material on functional programming, dataflow and multithreaded architectures, parallel processing, and high-level languages for ASIC systems design. $\mathrm{He}$ is a member of the ACM, the IEEE, and the IFIP WG 2.8 on Functional Programming.

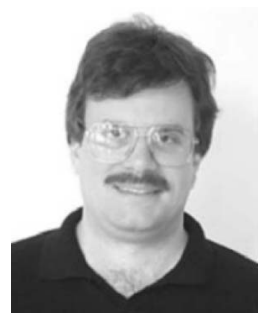

James $\mathrm{M}$. Rehg received the $\mathrm{PhD}$ degree from Carnegie Mellon University in 1995. From 1996 to 2001 , he led the computer vision research group at the Cambridge Research Laboratory of the Digital Equipment Corporation, which was acquired by Compaq Computer Corporation in 1998. In 2001, he joined the faculty of the College of Computing at the Georgia Institute of Technology, where he is currently an associate professor. His research interests include computer vision, machine learning, human-computer interaction, computer graphics, and distributed computing.

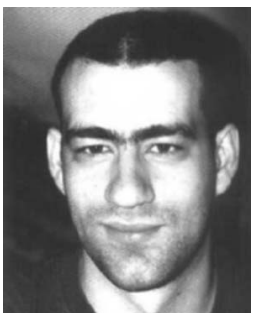

Yavor Angelov received the BA degree in computer science from the American University in Bulgaria, and the MS degree in computer science from Georgia Tech. He is a doctoral student in the College of Computing, Georgia Tech. His interests are in parallel and distributed computing.

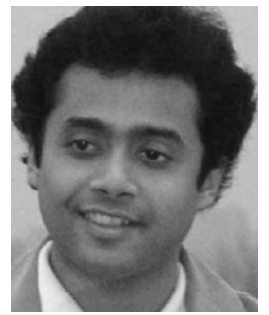

Arnab Paul received the undergraduate degree in computer science and engineering from Jadavpur University, India, and the Master's degree from the Indian Institute of Science, Bangalore. He is currently a $\mathrm{PhD}$ student at College of Computing, Georgia Tech, Atlanta. His interests include distributed and parallel computing, fault tolerant compuitng and storage systems, and information and coding theory. 


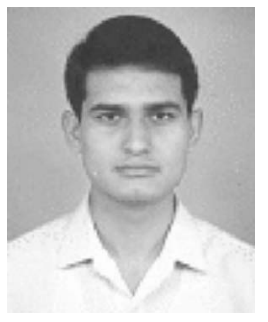

Sameer Adhikari has been a PhD student in the College of Computing at the Georgia Institute of Technology since 1998. He received the BTech degree from the Indian Institute of Technology, Kharagpur, India. His research interest is experimental computer systems, primarily distributed systems for pervasive computing.

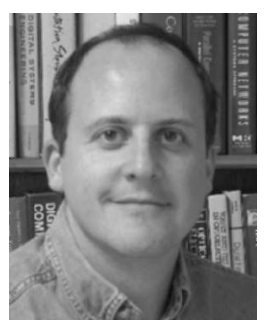

Kenneth M. Mackenzie received the SB, SM and $\mathrm{PhD}$ degrees from the Department of Electrical Engineering and Computer Science at the Massachusetts Institute of Technology in 1990, 1990, and 1998, respectively. From 1998 to 2003, he was an assistant professor in the College of Computing at the Georgia Institute of Technology, where he received a US National Science Foundation CAREER award in 1999. In 2003, he joined the technical staff of Reservoir Labs, New York, New York. His research interests are in the areas of parallel systems, embedded computing, and compilers.

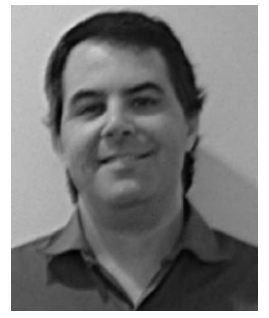

Nissim Harel received the BSc degree in electrical engineering from the Technion in Haifa, Israel, in 1990, and the MS degree in computer science, and the MSc degree in electrical engineering from the Georgia Institute of Technology in 1998 and 1999, respectively. $\mathrm{He}$ is a PhD student in the College of Computer Science at the Georgia Institute of Technology. $\mathrm{He}$ also cofounded in 2000, ClickFox, LLC, which develops and provides a decision support tool that helps optimizing interfaces of information systems, in general, and Web sites and IVR systems, in particular.

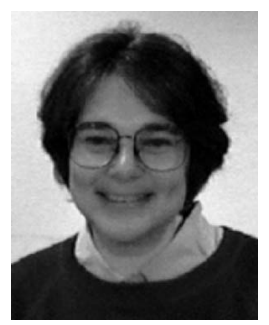

Kathleen Knobe worked at Massachusetts Computer Associates (AKAC ompass) for more than 10 years, where she worked on Fortran compilers for Alliant, Thinking Machines, and MasPar. She subsequently returned to complete her education and received the $\mathrm{PhD}$ degree from MIT in 1997, with Professor Wm. Dally. She currently works at Cambridge Research Lab (Digital/Compaq/HP). Her research interests include compilation, runtime systems, streaming, parallelism, and models of computation. She is currently investigating a new model of parallel computation based on streaming.

$\triangleright$ For more information on this or any other computing topic, please visit our Digital Library at http://computer.org/publications/dlib. 